\title{
MicroRNA-21 promotes the cell proliferation, invasion and migration abilities in ovarian epithelial carcinomas through inhibiting the expression of PTEN protein
}

\author{
YANHUI LOU ${ }^{1,2}$, XINGSHENG YANG ${ }^{2}$, FULING WANG $^{1}$, ZHUMEI CUI $^{1}$ and YU HUANG ${ }^{1}$ \\ ${ }^{1}$ Department of Gynecology, Affiliated Hospital of Medical College, Qingdao University, Qingdao; \\ ${ }^{2}$ Department of Obstetrics and Gynecology, Qilu Hospital of Shandong University, Jinan, Shandong, P.R. China
}

Received July 5, 2010; Accepted August 18, 2010

DOI: 10.3892/ijmm_00000530

\begin{abstract}
Ovarian cancer, especially epithelial ovarian cancer (EOC), which accounts for $90 \%$ of ovarian cancer, continues to be the leading cause of death among gynecological malignancies. However, the factors associated with its malignant biological behavior are still poorly understood. Accumulating evidence suggests that microRNAs (miRNAs), regulating diverse biological processes, may play an important role in tumorigenesis and development. miR-21 has been frequently observed to be aberrantly overexpressed in various tumors. Using real-time PCR, we confirmed that miR-21 was significantly overexpressed in human EOC tissues and cell lines. The overexpression of miR-21 correlated with histological differentiation, clinicopathological stage, and lymph node metastasis, and we showed that knockdown of miR-21 by an inhibitor caused a significant reduction in cell proliferation and decrease in cell migration and invasion abilities. Furthermore, we demon-strated that knockdown of miR-21 significantly increased the expression of PTEN, a known tumor suppressor in ovarian cancer. Collectively, our findings suggest miR-21 may be important in the initiation and progression of EOC as an oncomiR, likely through regulating PTEN.
\end{abstract}

\section{Introduction}

miRNAs are a class of small non-coding RNA molecules, 20-22 nucleotides in length, that regulate gene expression by direct cleavage of the targeted mRNAs or by inhibiting translation through perfect or nearly perfect complementarity

Correspondence to: Dr Yanhui Lou, Department of Gynecology, Affiliated Hospital of Medical College, Qingdao University, 16\# Jiangsu Road, Qingdao, Shandong 266001, P.R. China E-mail:1yh7497@163.com

Dr Xingsheng Yang, Department of Obstetrics and Gynecology, Qilu Hospital of Shandong University, Jinan, Shandong 250012, P.R. China

E-mail: yxsqilu_1@163.com

Key words: microRNA-21, epithelial ovarian carcinoma, PTEN, real-time RT-PCR, OVCAR-3 cells to targeted mRNAs at the 3'-untranslated regions (UTRs). Previous studies show that more than half of the miRNAs can be aligned to genomic fragile sites or regions associated with cancers (1), which indicates that miRNAs might play an important role in cancer pathogenesis, development, and metastasis. Aberrant expression of miRNA has been reported in various tumors such as breast, lung, liver, colon and gastric cancer indicating that there is a close correlation between miRNAs and human malignancy (2-5).

Many studies suggest that miR-21 is aberrantly overexpressed and function as an oncomiR in diverse tumors, including ovarian cancer (6-8). How miR-21 affects cancer development and progression is still not well understood. Si et al (8) reported that mir-21 promotes growth of the breast cancer cell line MCF-7 both in vitro and in vivo, which may be due to the ability of miR-21 to suppress the expression of the tumor suppressor PTEN and TPM1 (9). Meng et al (10) found that the overexpression of miR-21 stimulates increased migration and invasion through targeting the PTEN tumor suppressor gene in hepatocellular carcinoma cells, suggesting that PTEN might be a target gene for miR-21. However, the role and relevant pathway of miR-21 in ovarian carcinogenesis is largely unknown. Frequent inactivition of PTEN has been implicated in ovarian cancer $(11,12)$. However, PTEN mutations, promoter methylation and LOH at PTEN locus are not main causes for PTEN inactivation $(13,14)$. The mechanisms of down-regulation of PTEN expression in ovarian cancer remain unclear.

In our present study, we observed a significant overexpression of miR-21 in epithelial ovarian cancer (ECO) tissues and OVCAR-3 cell lines. The effect of miR-21 on cell proliferation, migration, and invasion ability were further investigated. Moreover, we found PTEN was negtively correlated with miR-21 in EOC tissues and significantly regulated by miR-21 OVCAR-3 cell lines. Our results suggested that miR-21 may function as an oncomiR in tumorigenesis and progression in ovarian cancer by targeting PTEN expression.

\section{Materials and methods}

Cell lines, patients, and tissue samples. OVCAR-3 cells (low grade serous ovarian papillary adenocarcinoma) were 
Table I. Primers used for real-time RT-PCR assay.

\begin{tabular}{lll}
\hline Target & Primer & \multicolumn{1}{c}{ Sequence } \\
\hline hsa-miR-21-1 $^{\text {a }}$ & RT & 5'GTCGTATCCAGTGCGTGTCGTGGAGTCGGCAATTGCACTGGATACGACTCAACATC3' \\
& F primer & 5'GCGGTAGCTTATCAGACTGA3' \\
& R primer & 5'TGCGTGTCGTGGAGTC3' \\
hsa-miR-21-2 & RT & 5'GTCGTATCCAGTGCGTGTCGTGGAGTCGGCAATTGCACTGGATACGACACAGCCCA3' \\
& F primer & 5'GCGGCAACACCAGTCGATG3' \\
& R primer & 5'TGCGTGTCGTGGAGTC3' \\
& F primer & 5'GCTTCGGCAGCACATATACTAAAAT3' \\
U6 & R primer & 5'CGCTTCACGAATTTGCGTGTCAT3' \\
\end{tabular}

ahsa-miR-21-1, primers for expression of miR-21 both in frozen EOC tissues and in OVCAR-3 cells; bhsa-miR-21-2, primers only for expression of miR-21 in OVCAR-3 cells.

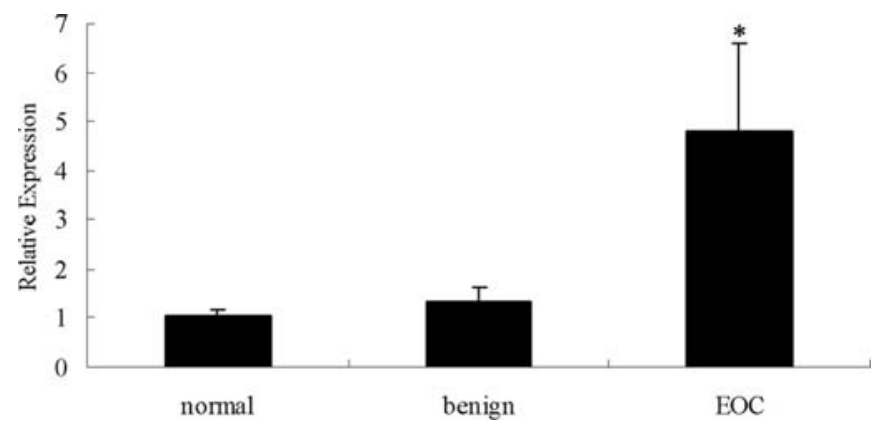

Figure 1. Relative expression of miR-21 in epithelial ovarian cancer, benign ovarian cysts, and normal ovarian tissue. Relative expression of miR-21 was detected with real-time RT-PCR and the relative amount of miR-21 was described using $2^{-\Delta \triangle C T}$. A T-test was used to analyze the data. miR-21 was significantly overexpressed in EOC $\left({ }^{*} \mathrm{p}<0.01\right)$.

purchased from the Shanghai cell library of Academia Sinica Type Culture Collection. A total of 87 tissue samples, including 48 epithelial ovarian cancer tissues, 24 benign ovarian epithelial cysts, and 15 normal ovarian tissue sections were collected by surgery at the Affiliated Hospital of Qingdao University Medical College. Ethical approval was obtained from the Institutional Review Boards, and informed consent for the use of tissues was obtained for all individuals. One part was snap-frozen in liquid nitrogen and stored at $-80^{\circ} \mathrm{C}$, and the other part was fixed in $10 \%$ formalin. The histopathological diagnoses were determined using the WHO criteria, and the tumor histotypes included 28 serous, 14 mucinous, and six endometrioid carcinomas. All tumors were staged according to the International Federation of Gynecology and Obstetrics standards (FIGO). Stage breakdown was: $n=5$ for stage I, $n=11$ for stage II, $n=23$ for stage III, and $n=9$ for stage IV. None of the patients received chemotherapy, radiotherapy, or hormone therapy before the surgical operation.

Construction of a short-hairpin RNA targeted miR-21 expression vector. A short-hairpin RNA specifically targeting miR-21 was constructed as follows: From the microbase databank (http://www.mirbase.org/cgi-bin/mirna), we obtained two mature fragment sequences for miR-21 and synthesized the following short anti-mir-21 RNA oligonucleotides: miR-21-1: 5'-gatccCAACATCAGTCTGATAAGCttcaag agaGCTTATCAGACTGATGTTGTTTTTTg-3'; miR-21-2: 5'-gatccCAGCCCATCGACTGGTGTTAtcaagagaAAC ACCAGTCGATGGGCTGTTTTTTg-3'. A control sequence that was not specific for miR-21 was also constructed: miR-21neg: 5'gatccGGCGCATAAGAAGCATATAttcaagagaTAT ATGCTTCTTATGCGCCTTTTTTg-3'. All the sequences were evaluated for sequence specificity by a BLAST search and did not show homology to other known genes. Annealed oligonucleotides were ligated with vector Linear pSIRENRetroQ, resulting in three recombinants: pSIREN-miR-21-1, pSIREN-miR-21-2, and pSIREN-miR-21-Neg. The recombinants were identified by restriction endonuclease analysis and DNA sequencing, respectively.

Cell culture and transfection. OVCAR-3 cells were cultured in McCoy's 5A medium with 10\% fetal bovine serum, $100 \mathrm{U} /$ $\mathrm{ml}$ penicillin, and $100 \mu \mathrm{g} / \mathrm{ml}$ streptomycin, and incubated at $37^{\circ} \mathrm{C}$ in a humidified atmosphere containing $5 \% \mathrm{CO}_{2}$. Twentyfour hours prior to transfection, cells were seeded in 6-well plates at a density of $5 \times 10^{5}$ cells/well and grown to $60 \%$ confluence. OVCAR-3 cells were transfected using the Lipofectamine 2000 reagent according to the manufacturer's instructions. Briefly, pDsRed $(7 \mu 1 / 100 \mu 1)$ was mixed with pSIREN-miR-21 recombinants $(3.5 \mu \mathrm{l} / 100 \mu \mathrm{l})$. Diluted Lipofectamine 2000 reagent (1:100) $(100 \mu 1)$ was added to the mixture of pSIREN-miR-21 recombinants $(100 \mu 1)$, respectively. After incubation for $15 \mathrm{~min}$ at room temperature, $200 \mu 1$ of transfection mixture was added to each well, resulting in a siRNA concentration of $1 \mu \mathrm{g} / \mu 1$.

Isolation of total RNA. RNA was extracted from cell lines and frozen tissue samples using the TRIzol reagent (Invitrogen Life Technologies) according to the manufacturer's instructions. The dissolved RNA was stored at $-70^{\circ} \mathrm{C}$ before use. RNA quality was assessed with a NanoDrop1000 spectrophotometer (NanoDrop Technologies). 
Real-time RT-PCR for miR-21 quantification. We designed gene-specific looped primers for RT-PCR (listed in Table I). Reverse transcription of RNA used $1 \mu \mathrm{g}$ total RNA in a Gene Amp PCR System 9700 (Applied Biosystems) in a total volume of $20 \mu \mathrm{l}$. The reaction proceeded for $30 \mathrm{~min}$ at $16^{\circ} \mathrm{C}$, $42 \mathrm{~min}$ at $42^{\circ} \mathrm{C}, 5 \mathrm{~min}$ at $85^{\circ} \mathrm{C}$ and the cDNA was stored at $-20^{\circ} \mathrm{C}$. Real-time PCR was performed using SYBR Green I (Invitrogen) detection chemistry on a Rotor-Gene 3000 realtime PCR machine (Corbett Research). The reactions were amplified for an initial 5-min denaturation step at $95^{\circ} \mathrm{C}$, followed by 40 cycles of $10 \mathrm{sec}$ at $95^{\circ} \mathrm{C}, 20 \mathrm{sec}$ at $60^{\circ} \mathrm{C}, 20 \mathrm{sec}$ at $72^{\circ} \mathrm{C}$ and $20 \mathrm{sec}$ at $78^{\circ} \mathrm{C}$ for collecting fluorescence. Realtime PCR for the U6 gene was performed in the same manner as the corresponding primers. All the reactions were typically run in duplicate three times, simultaneously. The cycle number at which the fluorescent signal in each reaction tube crossed a threshold (CT) was determined for miR-21 and the relative amount of miR-21 in tumor tissue to that in control group was described using the equation $2^{-\Delta \triangle \mathrm{CT}}$, where $\Delta \triangle \mathrm{CT}=$ (CT miR-21 - CT U6) tumor - (CT miR-21 - CT U6) control group.

Immunohistochemistry. PTEN was detected using a mouse monoclonal antibody (ZM 0221, 1:100, Zhongshan Technology, Bingjin, China). After antigen preparation and blocking in $10 \%$ goat serum, the specimens were incubated overnight at $4^{\circ} \mathrm{C}$ with primary antibodies (100X diluted). After washing, the slides were incubated with rabbit biotinylated antibody for $30 \mathrm{~min}$ at $37^{\circ} \mathrm{C}$. Immunostaining was revealed with the SP (streptavidin peroxidase) system (Zhongshan Technology) using diaminobenzidine as a chromogen. The slides were counter-stained with hematoxylin before mounting. PBS replaced the primary antibody for the negative control and a known breast cancer slide was used as the positive control. For the assessment of staining, five fields were randomly selected and their scores were averaged. The percentage of tumor cytoplasm showing positive staining for PTEN was recorded and scored as follows: a score of 0 for $0 \%$ staining, 1 for staining of $<10 \%, 2$ for $10-50 \%, 3$ for $50-80 \%$, and 4 for $>80 \%$. The average intensity, corresponding to the presence of negative, weak, intermediate, and strong staining, was given a score from 0 to 3 , respectively. The positive cell score was added to the average intensity to form an additive score for semiquantitative assessment as follows: regardless of the intensity of staining, a positive cell score $<10 \%$ for negative, a score of 3 for,$+ 4-5$ for,$++ 6-7$ for +++ .

Western blotting. Cells were washed twice with ice-cold PBS and lysed on the culture dishes using lysis buffer $(20 \mathrm{mM}$ Tris $\mathrm{pH} 7.5,150 \mathrm{mM} \mathrm{NaCl}, 1 \%$ Triton $\mathrm{X}-100,10 \%$ protease inhibitors). The protein concentration was determined using the BCA method and $20 \mu \mathrm{g}$ of each sample was separated by SDS-PAGE (10 and 4\%) and transferred to PVDF membranes. Nonspecific binding sites were blocked by incubating with $5 \%$ BSA solution for $1 \mathrm{~h}$ at room temperature. The membranes were then incubated with rabbit antihuman PTEN antibody (1:6000) and rabbit anti- $\beta$-actin polyclonal antibody $(1: 1000)$ overnight at $4^{\circ} \mathrm{C}$. Anti-rabbit secondary antibodies conjugated to horseradish peroxidase were used at a dilution of 1:2000. The protein was visualized using ECL Western blotting detection reagents and analyzed by scanning densitometry by Smart View Analysis Software (Shanghai Furi Science \& Technology Co. Ltd.).

Cell proliferation assays. Cell proliferation was assessed using an MTT assay. Cells $\left(5.0 \times 10^{3}\right.$ cells/well) were seeded on 96-well plates. At a series of time points, $10 \mu 1$ of MTT was added to each well and the cells were incubated at $37^{\circ} \mathrm{C}$ for $4 \mathrm{~h}$. Then, $100 \mu \mathrm{l}$ dimethylsulfoxide of the dimethyl sulfoxide was added to each well. The plates were shaken for $30 \mathrm{sec}$. The optical density (OD) was measured at $570 \mathrm{~nm}$ by using a microplate reader (Bio-Rad Model 680, Richmond, CA, USA).

Scratch-wound assay. The cells were plated onto six-well plates at a concentration of $1 \times 10^{5}$ cells per well. Cell monolayers were carefully wounded by scratching with a sterile plastic pipette tip. The cells were washed twice with cooled PBS and incubated for $24 \mathrm{~h}$. For each wound, 5 fields of excursion were photographed at 0 , and $48 \mathrm{~h}$ after treatment.

Cell invasion assay. Cells $\left(2.5 \times 10^{4}\right.$ cells per well $)$ were seeded onto the top chamber of a 24 -well matrigel-coated polyethylene terephthalate membrane inserts with $8 \mu \mathrm{m}$ pores (Corning). The bottom chamber was filled with $0.6 \mathrm{ml}$ McCoy's 5A with $10 \%$ FBS as a chemoattractant. After incubation for $24 \mathrm{~h}$, the filter membrane was fixed with $100 \%$ methanol and stained with hematoxylin and eosin. The degree of invasiveness was quantified by counting the number of cells in 10 random fields of view per filter, using x400 magnification. Data obtained from three separate inserts are shown as mean values.

Statistical analysis. Data analyses were performed with the statistical software package SPSS 13.0. The Student t-test was used to assess miR-21expression between different groups. The correlations between miR-21 or PTEN expression and the clinicopathological features were assessed with a Chisquare $\left(\chi^{2}\right)$ test when appropriate. A Spearman rank correlation coefficient test was used for the correlation between miR-21 with PTEN expression. The level of statistical significance was set at $\mathrm{p}<0.05$.

\section{Results}

Expression of miR-21 in different ovarian tissue samples. The $C_{T}$ values of miR-21 in ovarian epithelial carcinomas were lower than those in benign ovarian cysts and normal ovarian tissues. The results shown in Fig. 1 indicate that, among the 48 ovarian cancer samples analyzed, the relative expression of miR-21 $\left(2^{-\triangle \Lambda C T}=4.849 \pm 1.813\right)$ was significantly up-regulated by 4-fold or more compared with the benign ovarian cysts $(1.133 \pm 0.291)$ and normal ovarian tissues $(1.057 \pm 0.126)(\mathrm{p}<0.01$, respectively $)$. No differences were observed in miR-21 relative expression between benign ovarian cysts and normal controls.

miR-21 overexpression in relation to clinicopathological features. As shown in Table II, the relative expressions of miR-21 were significantly higher in advanced stage and 


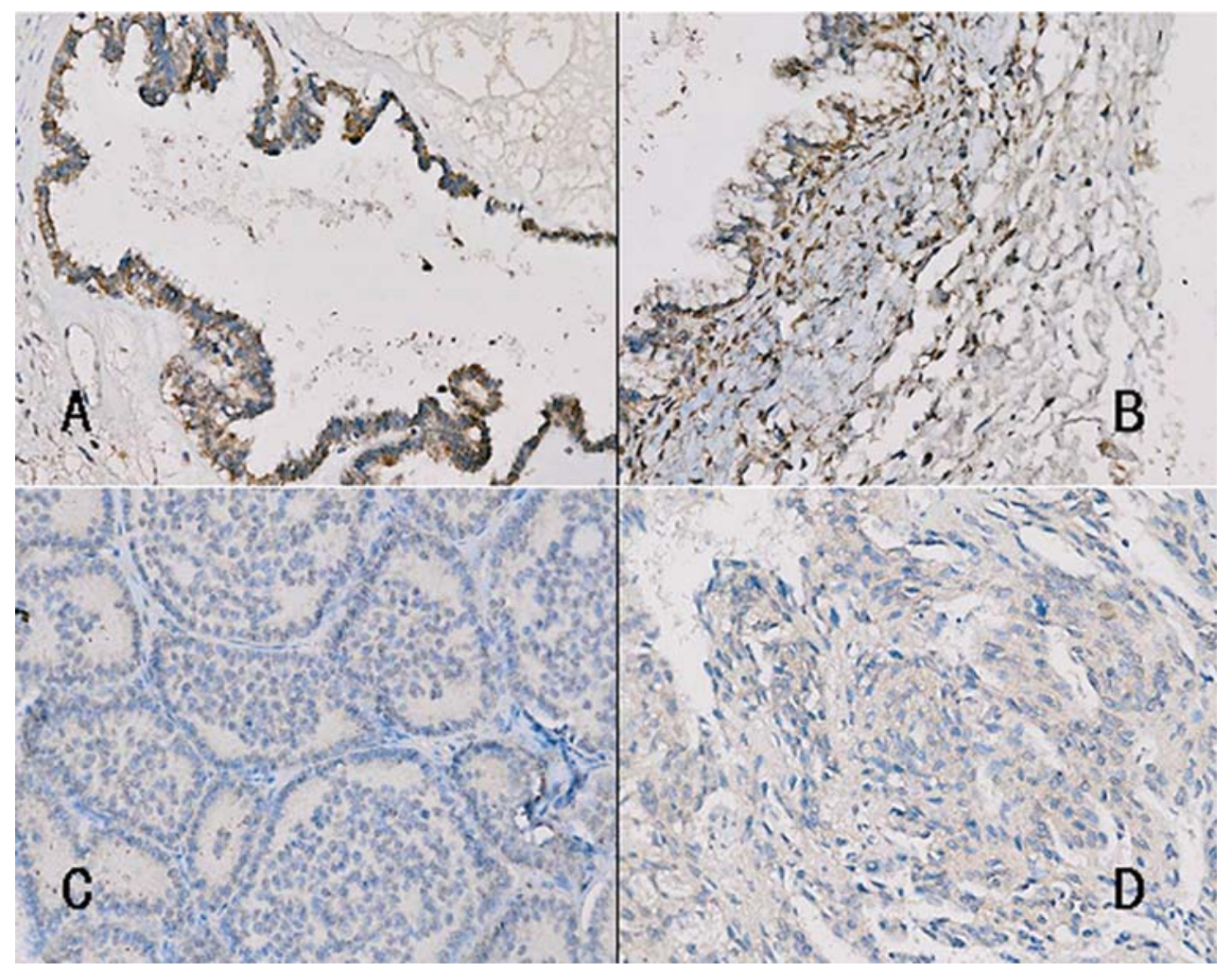

Figure 2. Representative immunohistochemistry of PTEN expression in epithelial ovarian cancer. The expression of PTEN was generally localized in the cytoplasm of the tissue specimens (A) positive staining in serous cystadenoma and (B) mucinous cystadenoma (+++, x200); (C) negative expression in mucinous ovarian cancer (x200); (D) weak staining in serious ovarian cancer (x200).

Table II. miR-21 and PTEN expression in relation to the clinicopathological features in epithelial ovarian cancer.

\begin{tabular}{|c|c|c|c|c|c|}
\hline Parameter & Cases (n) & miR-21 (mean $\pm \mathrm{SD})$ & p-value & PTEN (\% positive cases) & p-value \\
\hline \multicolumn{6}{|l|}{ FIGO stage } \\
\hline I, II & 16 & $3.341 \pm 0.254$ & & $12(75.00 \%)$ & \\
\hline III, IV & 32 & $5.603 \pm 1.787$ & $\mathrm{p}<0.01$ & $8(25.00 \%)$ & $\mathrm{p}=0.001$ \\
\hline \multicolumn{6}{|l|}{ Grade } \\
\hline $\mathrm{G} 1, \mathrm{G} 2$ & 20 & $3.845 \pm 0.680$ & & $17(85.00 \%)$ & \\
\hline G3 & 28 & $7.057 \pm 1.552$ & $\mathrm{p}<0.01$ & $3(10.71 \%)$ & $\mathrm{p}=0.000$ \\
\hline \multicolumn{6}{|l|}{ Lymph node } \\
\hline Negative & 36 & $4.100 \pm 1.070$ & & $18(50.00 \%)$ & \\
\hline Positive & 12 & $7.095 \pm 1.728$ & $\mathrm{p}<0.01$ & $2(16.66 \%)$ & $\mathrm{p}=0.043$ \\
\hline \multicolumn{6}{|l|}{ Grade } \\
\hline Serous & 28 & $4.891 \pm 1.636$ & & $12(42.85 \%)$ & \\
\hline Mucinous & 14 & $4.904 \pm 2.278$ & & $6(42.85 \%)$ & \\
\hline Endometrioid & 6 & $4.441 \pm 0.892$ & $\mathrm{p}>0.05$ & $2(33.33 \%)$ & $\mathrm{p}=0.907$ \\
\hline
\end{tabular}

T-test and Chi-square $\left(\chi^{2}\right)$ test were used to analyze the data. $\mathrm{p} \leq 0.05$ was considered as significant. Both the expression of miR-21 and PTEN correlated with the stage, grade and lymph node positivity in ovarian cancer.

grade 3 tumor samples compared to the early stage and grade 1 to 2 tumor samples $\left(2^{-\Delta \Delta C T}=5.603 \pm 1.787\right.$ vs . $3.341 \pm 0.254$ and $2^{-\triangle \Delta C T}=7.057 \pm 1.552$ vs. $3.845 \pm 0.680$, $\mathrm{p}<0.01$ respectively). The same differences were observed between the lymph node positive group and negative group $\left(2^{-\triangle \triangle C T}=7.095 \pm 1.728\right.$ vs. $\left.4.100 \pm 1.070, p<0.01\right)$. However, 


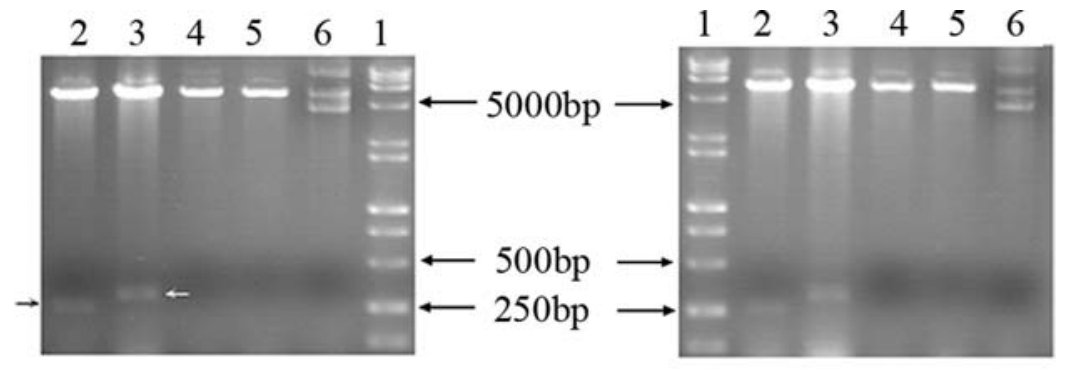

A

B

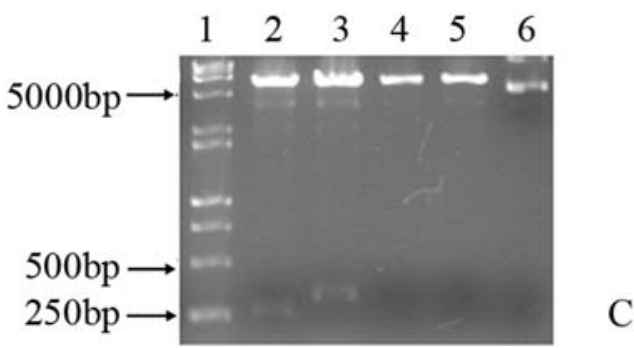

Figure 3. Identification of recombinant plasmid pSIREN-miR-21 by restriction endonucleases. (A) pSIREN-miR-21-1; (B) pSIREN-miR-21-2; (C) pSIRENmiR-21-neg. Lane 1, DNA Marker; lane 2, pSIREN-miR-21 digested by BamHI/BglII, yielding a fragment of 263 bp; lane 3, pSIREN-miR-21 digested by EcoRI/BglII, yielding a fragment of 322 bp; lane 4, pSIREN-miR-21 digested by BamHI; lane 5, pSIREN-miR-21 digested by EcoRI; lane 6, recombinant plasmid pSIREN-miR-21.

Table III. Immunohistochemical profiling of PTEN in epithelial ovarian cancers, benign ovarian cysts, and normal ovarian tissues.

\begin{tabular}{|c|c|c|c|c|c|c|c|}
\hline \multirow{2}{*}{$\begin{array}{l}\text { Group } \\
\text { (no. of cases) }\end{array}$} & \multicolumn{4}{|c|}{ Staining intensity } & \multirow[b]{2}{*}{ Positive cases $(\%)$} & \multirow[b]{2}{*}{$\chi^{2}$} & \multirow[b]{2}{*}{$\mathrm{p}$-value } \\
\hline & - & + & ++ & +++ & & & \\
\hline Normal $(n=15)$ & 0 & 2 & 6 & 7 & 100 & & \\
\hline Benign $(n=24)$ & 4 & 5 & 10 & 5 & 83.33 & 38.819 & 0.000 \\
\hline EOC $(n=48)$ & 28 & 12 & 8 & 0 & 41.66 & & \\
\hline
\end{tabular}

$0,+,++$ and +++ indicate negative, weak, moderate and strong immunostaining intensity, respectively. Of 48 ovarian cancer tissues, 20 $(41.66 \%)$ were positive for PTEN, mainly weakly positive expression, which was distinctly lower than that in begin ovarian cysts and normal control. Chi-square $\left(\chi^{2}\right)$ test was used to analyze the data. $\mathrm{p} \leq 0.05$ was considered as statistically significant.

we did not observe any significant differences in the three histotypes considered.

miR-21 overexpression is associated with decreased PTEN protein levels in ovarian cancer patients. As shown in Table III, 20 of $48(41.66 \%)$ ovarian cancer tissues were positive for PTEN, mainly weakly positive and expressed in the cytoplasm (Fig. 2), which was distinctly lower than that in begin ovarian cysts and normal controls. Moreover, eight of 32 (25\%) stage III-IV tissue samples were positive for PTEN, which was lower than that in stage I-II tissue samples $(12 / 16,75 \%)$. Three of $28(10.71 \%)$ grade 3 tissue samples and two of 12 (16.66\%) lymph node positive samples were positive for PTEN, which are obviously lower compared with grade1-2 samples $(17 / 20,85 \%)$ and lymph node negative samples $(20 / 36,55.55 \%)$ (Table II).

Seven cases were positive for PTEN among the 21 cases of ovarian cancer tissues with relatively low miR-21 expression (3.410 \pm 0.261$)$, but only six cases were positive for PTEN
Table IV. Correlation between miR-21 overexpression and PTEN in epithelial ovarian cancer.

\begin{tabular}{lcc}
\hline & \multicolumn{2}{c}{ PTEN } \\
\cline { 2 - 3 } $\operatorname{miR}-21(\mathrm{n}=48)$ & $\begin{array}{c}\text { Negative } \\
(\mathrm{n}=28)\end{array}$ & $\begin{array}{c}\text { Positive } \\
(\mathrm{n}=20)\end{array}$ \\
\hline $3.410 \pm 0.261(\mathrm{n}=21)$ & $7(33.3)$ & $14(66.6)$ \\
$5.967 \pm 1.711(\mathrm{n}=27)$ & $21(77.77)$ & $6(22.22)$ \\
\hline
\end{tabular}

Spearman rank correlation coefficient test was used for the data. The statistically significant negative correlation between miR-21 and PTEN was, $r=-0.447, \mathrm{p}<0.01$.

among the 27 cases of ovarian cancer tissues with relatively high miR-21 expression $(5.967 \pm 1.711)$. Spearman rank correlation analysis showed that PTEN expression was 
A

a

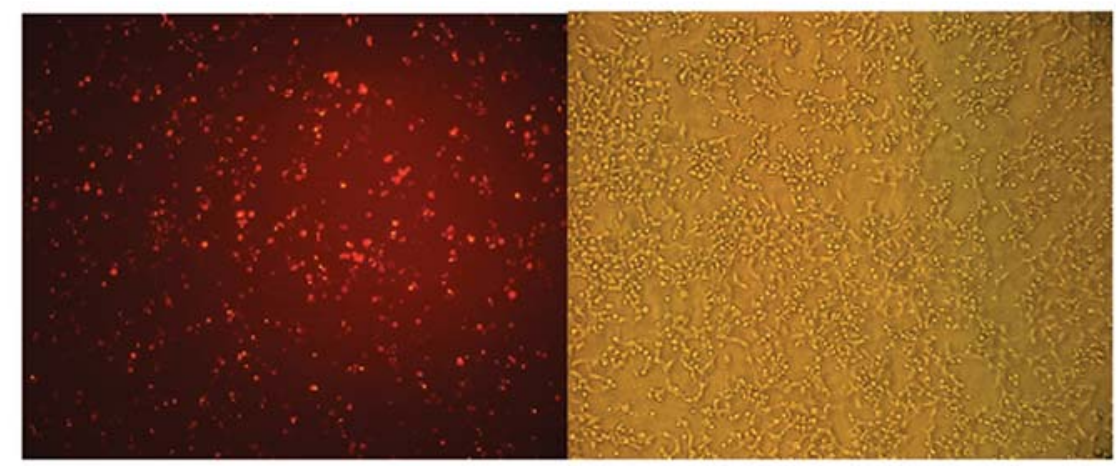

b

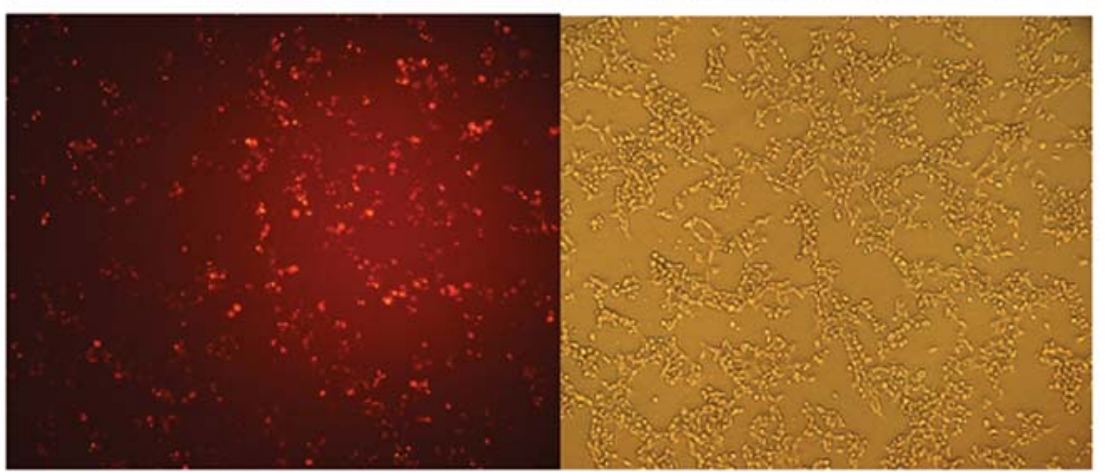

c

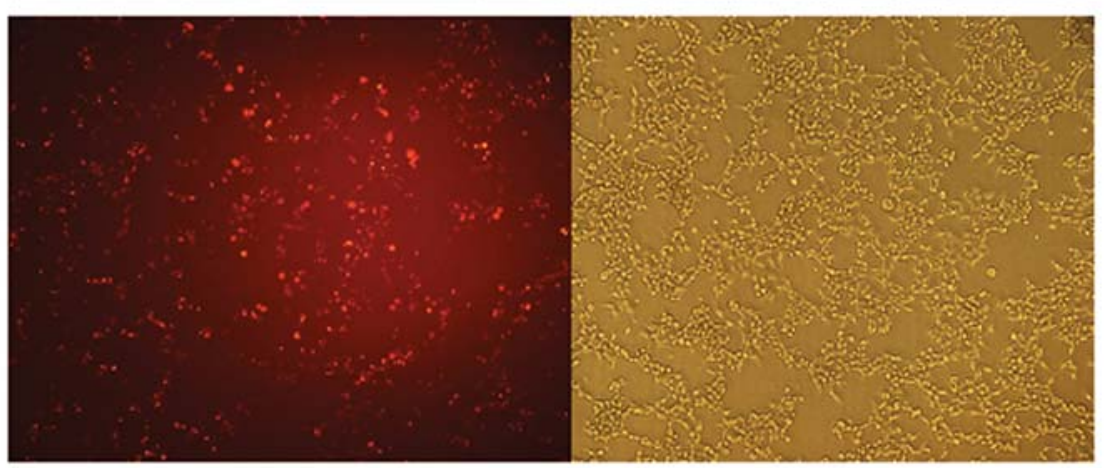

Figure 4. The expression of DsRed fluorescence in OVCAR-3 cells co-transfected with pDsRed and pSIREN-PSPRKs after $72 \mathrm{~h}$; (x100, exposure time: $500 \mathrm{msec}$, plus value: 2500). (A) Images of DsRed fluorescence; (B) Images of cells corresponding to (A). (a) Recombinant plasmid pDsRed co-transfected into OVCAR-3 cells with pSIREN-miR-21-neg; (b) recombinant plasmid pDsRed co-transfected into OVCAR-3 cells with pSIREN-miR-21-1; (c) recombinant plasmid pDsRed co-transfected into OVCAR-3 cells with pSIREN-miR-21-2.

negatively correlated with $\mathrm{miR}-21$ expression $(\mathrm{r}=-0.447$, $\mathrm{p}<0.01)($ Table IV).

Construction and identification of short-hairpin RNA expression vectors. When the annealed oligos (59 bp) were inserted downstream of the $\mathrm{H} 1$ promoter of the empty vector pSIREN-RetroQ, the recombinant vectors could be digested by $B a m \mathrm{HI} / B g l \mathrm{II}$ and $E c o \mathrm{RI} / B g l \mathrm{II}$ restriction enzymes to produce $263 \mathrm{bp}$ and $322 \mathrm{bp}$ fragments, respectively. When the recombinants were digested only by BamHI or EcoRI, no fragment was produced (Fig. 3). The recombinant vectors were then identified by DNA sequencing.

pSIREN-miR-21-1 and pSIREN-miR-21-2 efficiently suppressed miR-21 expression. Seventy-two hours after infection, all the pSIREN-miR-21 recombinants were mixed with pDsRed, respectively, and transfected into OVCAR-3 cells (Fig. 4). Decreased miR-21 expression was confirmed by real-time
RT-PCR. Data showed that miR-21 expression levels decreased significantly both in pSIREN-miR-21-1 and pSIREN-miR21-2 infected cells compared to pSIREN-miR-21-Neg infected cells and control group (Fig. 5).

miR-21 modulates PTEN expression in OVCAR-3 cells. To test whether PTEN was directly modulated by miR-21, Western blotting was used to detect PTEN expression in OVCAR-3 cells after transfection. The data showed that PTEN expression was increased in OVCAR-3 cells transfected by pSIREN-miR-21-1 and pSIREN-miR-21-2, respectively, compared with vector-control transfected cells (Fig. 6), indicating that inhibition of miR-21 can significantly increase PTEN expression in OVCAR-3 cell lines.

Down-regulation of miR-21 expression inhibits OVCAR-3 cell proliferation in vitro. Cell viability following transfection with pSIREN-miR-21 was tested by MTT assay after $72 \mathrm{~h}$. As 

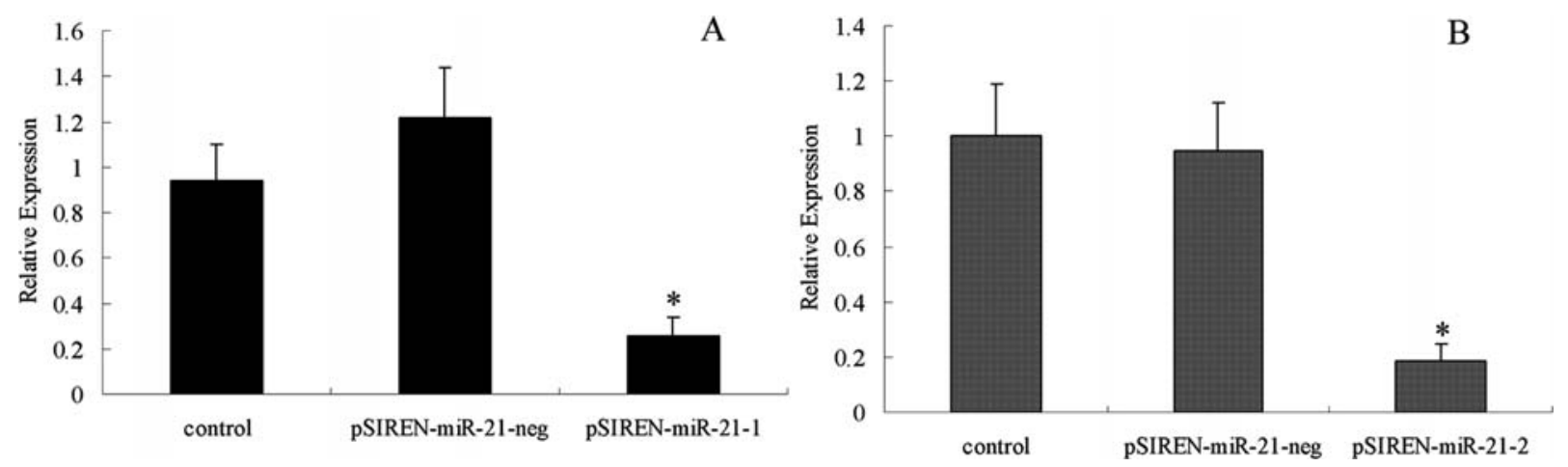

Figure 5. miR-21 expression was significantly downregulated in OVCAR-3 cells infected with pSIREN-miR-21-1 (A) and pSIREN-miR-21-2 (B). Relative expression of miR-21 was detected with real-time RT-PCR and the relative amount of miR-21 was described using $2^{-\Delta A C T}$. T-test was used to analyze the data compared with the pSIREN-miR-21-neg infected group and control group; ${ }^{*} \mathrm{p}<0.01$.

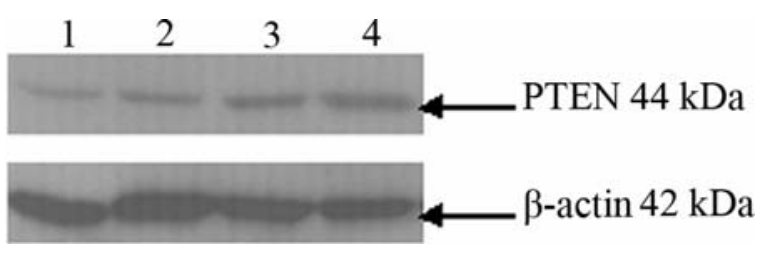

Figure 6. Expression of PTEN protein in infected OVCAR-3 cells by Western blot analysis. The protein levels of PTEN were much lower than those of pSIREN-miR-21 infected cells. Lane 1, Untransformed OVCAR-3 cells; lane 2, OVCAR-3 cells transfected with pSIREN-miR-21-neg; lane 3, OVCAR-3 cells transfected with pSIREN-miR-21-1; lane 4, OVCAR-3 cells transformed with pSIREN-miR-21-2.

showed in Fig. 7, there was no significant difference for light absorption values between pSIREN-miR-21-neg group and the control group. The inhibition rate in pSIREN-miR-21-1 and pSIREN-miR-21-2 group was higher than that in control group $(23.9,29.4$ and $9.03 \%$, respectively), indicating the cell proliferation ability was suppressed by transfection of pSIREN-miR-21-1 and -2.

miR-21 inhibits OVCAR-3 cells migration and invasion in vitro. In scratch-wound assay, we observed the scratched areas were mostly covered with cells both in blank control and negative control group, while there were fewer cells covered in pSIREN-miR-21-1 and pSIREN-miR-21-2 group (Fig. 8). To further quantitatively evaluate the effect of down-regulation of miR-21 in cell invasion, a transwell assay was performed. As shown in Fig. 9, the migrated cells in pIREN-miR-21-1 and pSIREN-miR-21-2 group were 40 \pm 3.56 and $29.5 \pm 2.38$ respectively, and the number was significantly lower than that in control group $(73 \pm 2.16)(\mathrm{p}<0.05)$. No significant difference was observed between control group and pSIRENmiR-21-net group $(69 \pm 1.83)$. The data indicate cell migration and invasion were significantly suppressed after being transfected with pSIREN-miR-21-1 and pSIREN-miR-21-2.

\section{Discussion}

Accumulated evidence has associated the aberrant expression of many miRNAs, including miR-21, with various cancers and certain oncogenes or tumor suppressors involved in

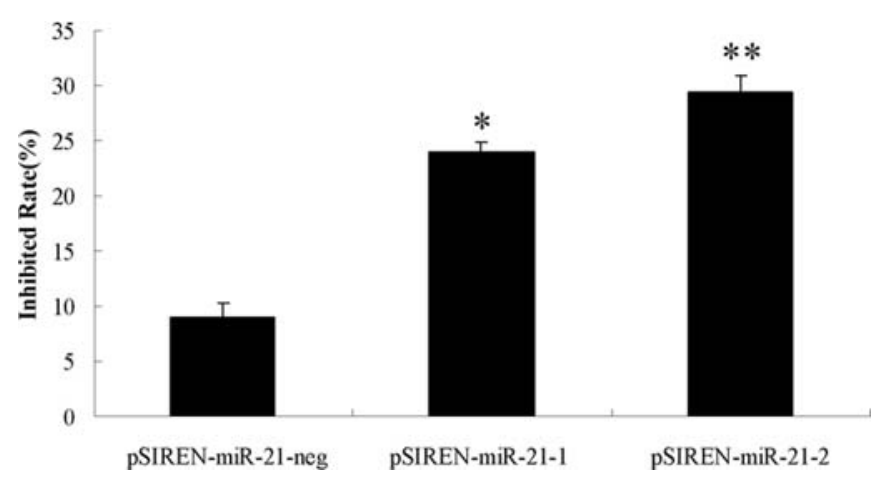

Figure 7. MTT assays for the evaluation of cell proliferation of OVCAR-3 transfected with pSIREN-miR-21. The inhibition rate in pSIREN-miR-21-1 and pSIREN-miR-21-2 group was $23.9 \%$ and $29.4 \%$, respectively, which were higher than negative control. Statistical analysis was performed using the Chi-square test; ${ }^{*} \mathrm{p}<0.05 ;{ }^{* *} \mathrm{p}<0.01$.

tumorigenesis $(15,16)$. However, the potential role and regulation mechanism of miRNAs in ovarian cancer are not well demonstrated. In this study, we showed that miR-21 was significantly up-regulated in epithelial ovarian cancer (EOC), suggesting that miR-21 may function as an oncogene in the pathogenesis of ovarian cancer.

Moreover, overexpression of miR-21 was closely correlated with advanced clinical stage, high grade, and lymph node metastases which are the main prognostic factors for ovarian cancer, implying miR-21 might be involved in the development and metastasis of cancer, and has a prognostic implication for EOC. We further demonstrated that knock-down of miR-21 could dramatically decrease cell proliferation, invasion and migration abilities in OVCAR-3 cells. Our data suggest that the aberrant expression of miR-21 directly affects invasive and metastatic potential of tumor cells and is implicated in the progression of ovarian cancer.

Previous studies have reported that miR-21 plays an important role not only in tumor growth but also in the invasion and metastasis by targeting multiple tumor/metastasis suppressor genes including PTEN, PDCD4, BCL-2 and TPM1 $(9,10,17)$. PTEN, well known for its role in tumor growth, invasion and metastasis, has been reported as a tumor suppressor gene in ovarian cancer $(13,18)$. Our study shows that $41.66 \%$ of EOC samples were positive for PTEN 

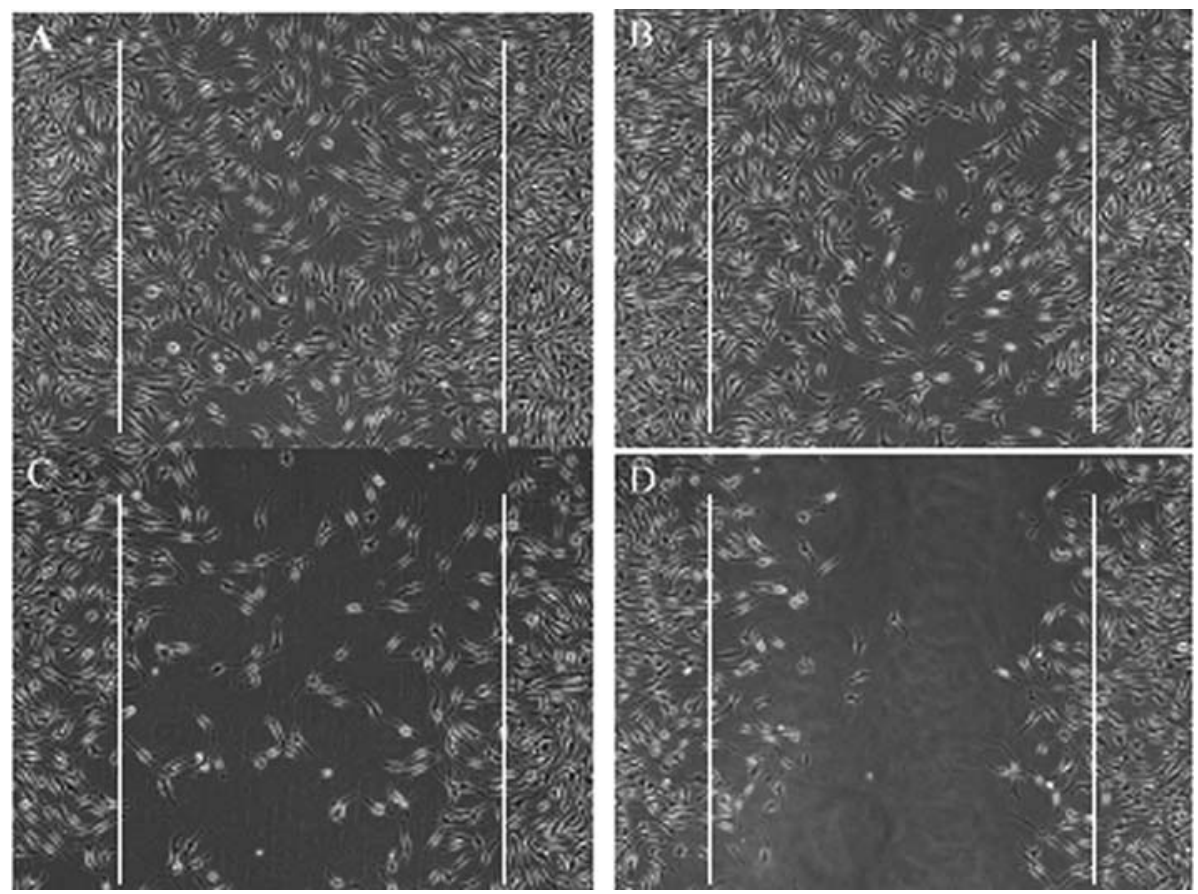

Figure 8. The effect of down-regulation of miR-21 on OVCAR-3 cell migration (48 $\mathrm{h}$ after transfection, $\mathrm{x} 100$ ). White line, $0 \mathrm{~h}$; (A) control group; (B) pSIREN-miR-21-net group; (C) pSIREN-miR-21-1 group; (D) pSIREN-miR-21-2 group.
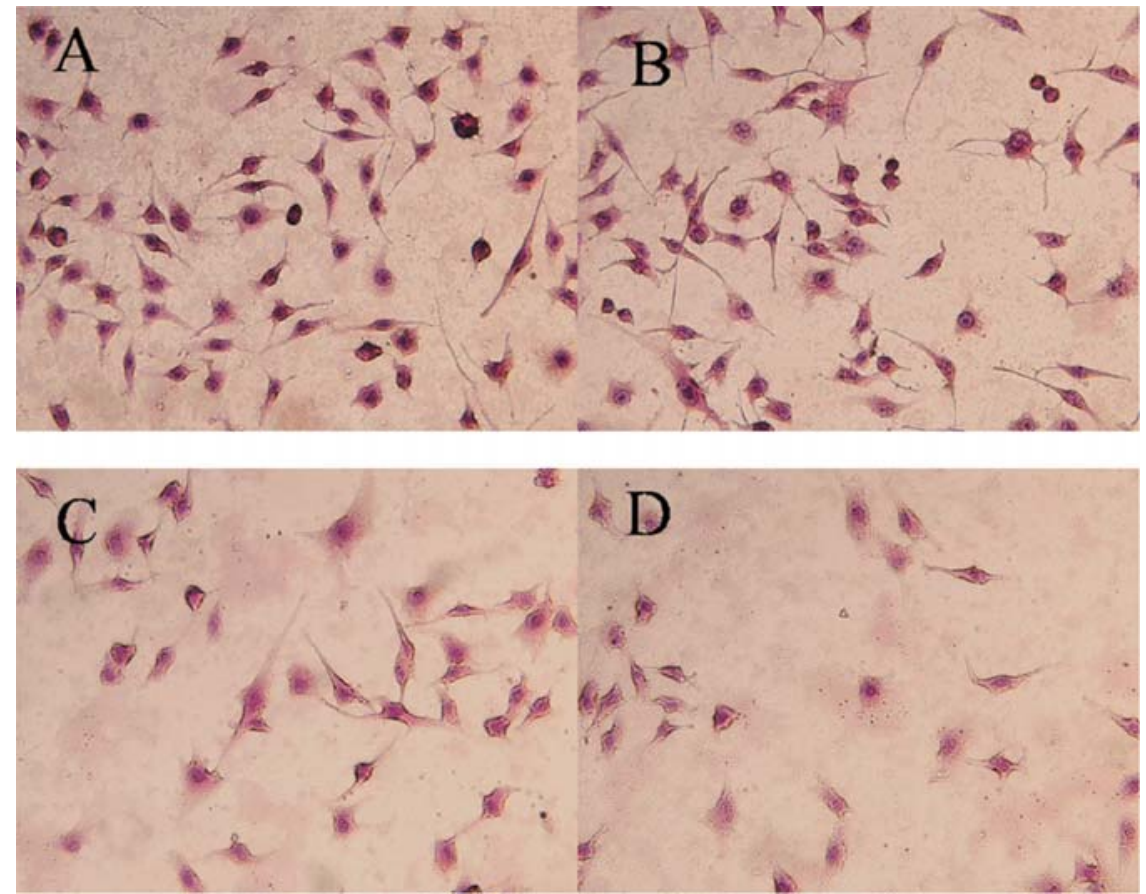

Figure 9. The effect of down-regulation of miR-21 on OVCAR-3 cell invasion (x400). (A) control group; (B) pSIREN-miR-21-net group; (C) pSIREN-miR21-1 group; (D) pSIREN-miR-21-2 group.

expression and $58.34 \%$ were negative that is consistent with previous studies $(18,19)$. PTEN expression show a negative correlation with the expression of miR-21 in EOC tissues. Furthermore, we demonstrated that inhibition of miR-21 can significantly increase PTEN expression in OVCAR-3 cell lines, suggesting epithelial ovarian cancer might enhance malignant biological behavior by inactivating PTEN genetically and up-regulating miR-21.

PTEN is capable of restricting growth and survival signals by limiting the activity of the phosphoinositide 3-kinase (PI3K) pathway. A decrease in functional PTEN causes constitutive activation of downstream components of the PI3K 
pathway including Akt, leading to tumor progression and metastasis (20). Thus, PTEN down-regulation by miR-21 may contribute to transformation and increased tumor cell survival (20). Meng et al (10) previously demonstrated that miR-21 can modulate gene expression directly at the PTEN 3'-UTR and that PTEN might be a potential target for miR-21. The inhibition of miR-21 using antisense oligonucleotides increased the expression level of PTEN in hepatocellular carcinoma cells (21). Zhu et al (22) also reported that miR-21 was implicated in invasion and metastases in breast cancer through decreasing the expression of PTEN. Therefore, our data together with other studies indicate that the mechanism by which miR-21 promotes tumor invasion and metastasis through down-regulating PTEN expression is a very important regulation pathway not only involved in ovarian cancer tumorigenesis and progression but also in other tumors.

Besides PTEN gene, several other genes have also been identified as targets of miR-21. For example, Chan and his colleagues reported that miR-21 was remarkably up-regulated in human glioblastoma and contributed to the malignant phenotype by blocking expression of PDCD4 gene (23). Zhu et al (9) identified a tumor suppressor TPM-1 as a potential target of miR-21. Therefore, the miR-21-mediated pathways appear to be very complex in various physiological and pathological process and tumorigenesis.

In conclusion, our study revealed a different expression profile for miR-21 in EOC tissue specimens compared with benign ovarian cysts and normal ovarian tissues. We show, for the first time, that overexpression of miR-21 is significantly correlated with negative expression of PTEN protein in EOC tissue specimens and cell lines. Aberrant expression of miR-21 can alter multiple biological processes of OVCAR-3 cells such as proliferation, migration, and invasion, probably through regulating PTEN. It is suggested that miR-21 functions as an ancomiR involved in EOC tumorigenesis and promotes invasion and metastasis. Therefore, targeting miR-21 may provide a better strategy for blocking tumor metastasis.

\section{Acknowledgements}

We would like to thank Dr Xingsheng Yang (Qilu Hospital of Shandong University, Jinan, China) for his critical reading of the manuscript and thank Mr. Haijing Su for excellent technical support.

\section{References}

1. Calin GA, Sevignani C, Dumitru CD, et al: Human microRNA genes are frequently located at fragile sites and genomic regions involved in cancers. Proc Natl Acad Sci USA 101: 2999-3004, 2004.

2. Yanaihara N, Caplen N, Bowman E, et al: Unique microRNA molecular profiles in lung cancer diagnosis and prognosis. Cancer Cell 9: 189-198, 2006.
3. Xi Y, Shalgi R, Fodstad O, Pilpel Y and Ju J: Differentially regulated micro-RNAs and actively translated messenger RNA transcripts by tumor suppressor p 53 in colon cancer. Clin Cancer Res 12: 2014-2024, 2006.

4. Wickramasinghe NS, Manavalan TT, Dougherty SM, Riggs KA, Li Y and Klinge CM: Estradiol downregulates miR-21 expression and increases miR-21 target gene expression in MCF-7 breast cancer cells. Nucleic Acids Res 37: 2584-2595, 2009.

5. Volinia S, Calin GA, Liu CG, et al: A microRNA expression signature of human solid tumors defines cancer gene targets. Proc Natl Acad Sci USA 103: 2257-2261, 2006.

6. Yang H, Kong W, He L, et al: MicroRNA expression profiling in human ovarian cancer: miR-214 induces cell survival and cisplatin resistance by targeting PTEN. Cancer Res 68: 425-433, 2008.

7. Slaby O, Svoboda M, Fabian P, et al: Altered expression of miR-21, miR-31, miR-143 and miR-145 is related to clinicopathologic features of colorectal cancer. Oncology 72: 397-402, 2007.

8. Si ML, Zhu S, Wu H, Lu Z, Wu F and Mo YY: miR-21-mediated tumor growth. Oncogene 26: 2799-2803, 2007.

9. Zhu S, Si ML, Wu H and Mo YY: MicroRNA-21 targets the tumor suppressor gene tropomyosin 1 (TPM1). J Biol Chem 282: 14328-14336, 2007.

10. Meng F, Henson R, Wehbe-Janek H, Ghoshal K, Jacob ST and Patel T: MicroRNA-21 regulates expression of the PTEN tumor suppressor gene in human hepatocellular cancer. Gastroenterology 133: 647-658, 2007.

11. Wu H, Cao Y, Weng D, et al: Effect of tumor suppressor gene PTEN on the resistance to cisplatin in human ovarian cancer cell lines and related mechanisms. Cancer Lett 271: 260-271, 2008.

12. Meng Q, Xia C, Fang J, Rojanasakul Y and Jiang BH: Role of PI3K and AKT specific isoforms in ovarian cancer cell migration, invasion and proliferation through the p70S6K 1 pathway. Cell Signal 18: 2262-2271, 2006.

13. Kurose K, Zhou XP, Araki T, Cannistra SA, Maher ER and Eng C: Frequent loss of PTEN expression is linked to elevated phosphorylated Akt levels, but not associated with p27 and cyclin D1 expression, in primary epithelial ovarian carcinomas. Am J Pathol 158: 2097-2106, 2001

14. Ho CM, Lin MC, Huang SH, et al: PTEN promoter methylation and $\mathrm{LOH}$ of 10q22-23 locus in PTEN expression of ovarian clear cell adenocarcinomas. Gynecol Oncol 112: 307-313, 2009.

15. Calin GA and Croce CM: MicroRNA signatures in human cancers. Nat Rev Cancer 6: 857-866, 2006.

16. Esquela-Kerscher A and Slack FJ: Oncomirs - microRNAs with a role in cancer. Nat Rev Cancer 6: 259-269, 2006.

17. Asangani IA, Rasheed SA and Nikolova DA: MicroRNA-21 (miR-21) post-transcriptionally downregulates tumor suppressor Pdcd 4 and stimulates invasion, intravasation and metastasis in colorectal cancer. Oncogene 27: 2128-2136, 2008.

18. Lee YK and Park NH: Prognostic value and clinicopathological significance of p53 and PTEN in epithelial ovarian cancers. Gynecol Oncol 112: 475-480, 2009.

19. Wang Y, Kristensen GB, Helland A, Nesland JM, BorresenDale AL and Holm R: Protein expression and prognostic value of genes in the erb-b signaling pathway in advanced ovarian carcinomas. Am J Clin Pathol 124: 392-401, 2005.

20. Sansal I and Sellers WR: The biology and clinical relevance of the PTEN tumor suppressor pathway. J Clin Oncol 22: 2954-2963, 2004.

21. Meng F, Henson R, Lang M, et al: Involvement of human micro-RNA in growth and response to chemotherapy in human cholangiocarcinoma cell lines. Gastroenterology 130: 2113-2129, 2006.

22. Zhu S, Wu H, Wu F, Nie D, Sheng S and Mo YY: MicroRNA-21 targets tumor suppressor genes in invasion and metastasis. Cell Res 18: 350-359, 2008.

23. Chan JA, Krichevsky AM and Kosik KS: MicroRNA-21 is an antiapoptotic factor in human glioblastoma cells. Cancer Res 65: 6029-6033, 2005. 